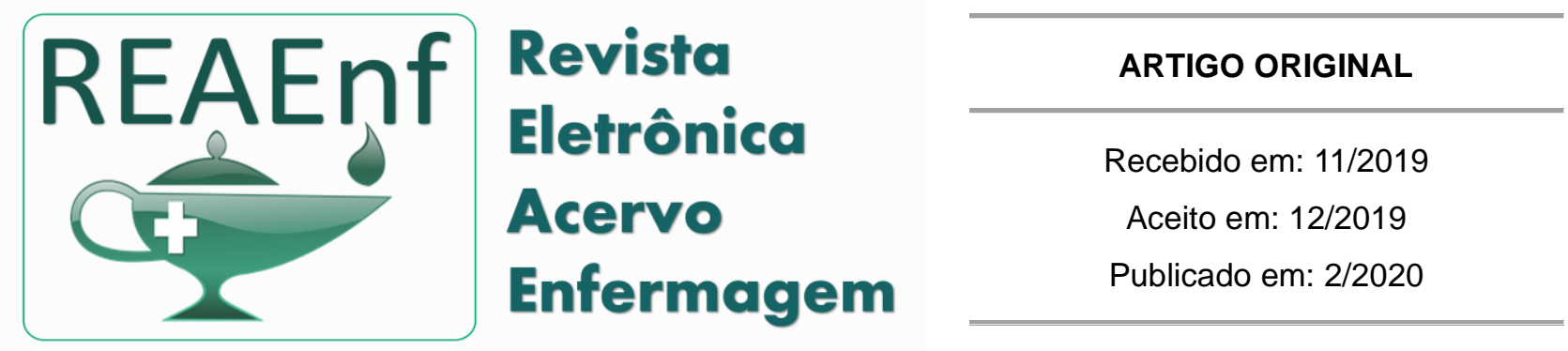

\title{
Ocorrência de febre maculosa brasileira no estado de minas gerais no período de 2013 a 2017
}

Occurrence of brazilian spotted fever in the state of Minas Gerais in the period 2013-2017

Ocurrência de la fiebre maculosa brasileña en el estado de Minas Gerais en el período 2013-2017

Luciano Gonçalves Lamas ${ }^{1 *}$, Jonathan Mendes de Castro ${ }^{1}$, Talles Vinícius de Castro Oliveira ${ }^{1}$, Enaile de Souza Proti ${ }^{1}$, Ranyelli Estefany da Costa Martins ${ }^{1}$, Marcela Alves Azevedo', Diego Azevedo Araújo ${ }^{1}$, Jacqueline Souza Dutra Arruda ${ }^{1}$, Carlos Henrique Wernersbach Guerra ${ }^{1}$, Wendel Jose Teixeira Costa².

Resumo: Esse trabalho tem por objetivo descrever a ocorrência da Febre Maculosa Brasileira nas macrorregiões do estado de Minas Gerais no período de 2013 a 2017. Trata-se de estudo transversal, descritivo, com delineamento de série temporal e utilização de dados de natureza secundária referentes à ocorrência de casos de Febre Maculosa, da população residente no Estado de Minas Gerais. Observou-se maior incidência de casos de Febre Maculosa Brasileira em homens, com menos de 8 anos de estudos, idade entre 20 e 59 anos, raça parda e negra, residentes nas macrorregiões Jequitinhonha, Leste do Sul e Sudeste, com alta mortalidade no período analisado. Estes dados evidenciam necessidade de atenção do poder público nas áreas mais incidentes, com foco na Atenção Básica, a fim de modificar essa realidade através de campanhas de conscientização, abordando a identificação de sinais e sintomas da doença, medidas de controle de vetores e prevenção da contaminação, a fim de reduzir a ocorrência de casos e principalmente de óbitos.

Palavras-chave: Febre Maculosa Brasileira, Perfil Epidemiológico, Incidência.

Abstract: This paper aims to describe the occurrence of brazilian spotted fever in the macroregions of the state of Minas Gerais from 2013 to 2017. This is a cross-sectional, descriptive study with time series delineation and use of secondary nature data to the occurrence of cases of brazilian spotted fever, of the population residing in the State of Minas Gerais. There was a higher incidence of brazilian spotted fever cases in men, with less than 8 years of schooling, aged between 20 and 59 years, black and brown race, living in the Jequitinhonha, South East and Southeast macroregions, with high mortality in the period analyzed. These data highlight the need for public attention in the most incident areas, focusing on Primary Care, in order to modify this reality through awareness campaigns, addressing the identification of signs and symptoms of the disease, vector control measures and prevention of the disease. contamination in order to reduce the occurrence of cases and especially deaths.

Keywords: Brazilian Spotted Fever, Health Profile, Incidence.

${ }^{1}$ Centro Universitário de Caratinga (UNEC), Caratinga-MG. *E-mail: lucianolamasg@hotmail.com 'Laboratório de Escrita Científica, Escola Superior de Ciências da Santa Casa de Misericórdia (EMESCAM), Vitória-ES. 
Resumen: Este artículo tiene como objetivo describir la aparición de la fiebre maculosa brasileña en las macrorregiones del estado de Minas Gerais de 2013 a 2017. Este es un estudio descriptivo de corte transversal con delineación de series de tiempo y uso de datos secundarios sobre la ocurrencia de casos de fiebre maculosa brasileña, de la población que reside en el estado de Minas Gerais. Hubo una mayor incidencia de casos de fiebre maculosa brasileña en hombres, con menos de 8 años de escolaridad, con edades entre 20 y 59 años, raza negra y marrón, que viven en las macroregiones de Jequitinhonha, Este del Sur y Sudeste, con alta mortalidad en el período analizado. Estos datos resaltan la necesidad de atención pública en las áreas más incidentes, enfocándose en la Atención Primaria, para modificar esta realidad a través de campañas de concientización, abordando la identificación de signos y síntomas de la enfermedad, medidas de control de vectores y prevención de la enfermedad. contaminación para reducir la ocurrencia de casos y especialmente de muertes.

Palabras clave: Fiebre Maculosa Brasileña, Perfil de Salud, Incidencia.

\section{INTRODUÇÃO}

A Febre Maculosa (FM) é uma Ricketsiose, que se configura com um grupo de doenças que se caracterizam como doenças febris agudas. No Brasil a Febre Maculosa é transmitida através da picada de carrapatos vetores da espécie Amblyomma cajennensee causada por bactérias da família Rickettsiae, visto que a espécie Rickettsia ricketsii possui uma maior evidência no Brasil e se configura como agente etiológico da Febre Maculosa Brasileira (DEL FIOL FS, et al., 2010; DIAS E e MARTINS AV, 1939).

A doença pode ser fatal se não for diagnosticada em tempo adequado e tratada corretamente. Além disso, a FM é a doença considerada como mais importante, sobre o ponto de vista epidemiológico, transmitida por carrapatos na América do Sul (HORTA MC, 2004; LABRUNA MB, 2009). No Brasil, estudos sobre a ecologia de vetores de febre maculosa foram demonstrados com três espécies de Rickettsiae associadas a focos de doenças: Rickettsia rickettsii , Rickettsia sp. cepa Mata Atlântica e Rickettsia parkeri (LABRUNA MB, 2009; SPOLIDORIO MG, et al., 2010; WECK B, et al., 2016).

Nas últimas décadas, foi observado um importante aumento do número de casos de FM no sudeste do Brasil, com destaque para os estados de São Paulo e Minas Gerais, considerados como áreas endêmicas para a enfermidade, sendo que a maioria dos casos foi confirmada por exames laboratoriais e a taxa média de mortalidade pela doença foi próximo a $30 \%$. A maioria desses casos foi confirmada apenas por técnicas sorológicas, sendo que a identificação específica da espécie Rickettsia não foi alcançada. No entanto, como esses casos eram clínicas e epidemiologicamente compatíveis com a FM, presumiu-se que o agente causal fosse Rickettsia rickettsii (LABRUNA MB, 2009).

A transmissão da bactéria Rickettsia, nos seres humanos, ocorre por meio da picada do vetor contaminado pela bactéria, através da saliva, uma vez que o mesmo se alimenta do sangue do hospedeiro. Pode ocorrer tanto nas fases ninfa e larva, quanto na fase adulta, conforme o período em que o vetor permanece aderido à pele, período este que varia entre 4 a 6 horas. $O$ carrapato infectado transmite a doença durante toda sua vida, que pode variar entre 18 e 36 meses (CHEN LF e SEXTON DJ, 2008).

O período de incubação da FM pode variar de 2 a 14 dias, a depender das condições fisiológicas do hospedeiro suscetível. Entre o segundo e o sexto dia apresentam-se as máculas (petéquias), apresenta quadro febril agudo, mialgia na forma intensa, cefaleia, edema em membros inferiores, oligúria, podendo evoluir para infecção generalizada caso o tratamento não seja rapidamente instaurado, podendo apresentar complicações no sistema circulatório, complicações pulmonares levando o paciente a coma e morte (MARTINS MEP, 2018).

A letalidade associa-se a alguns fatores importantes, dentre eles, um reconhecimento rápido e um tratamento adequado conforme a idade do paciente, uma vez que em crianças pode variar em $2 \%$ e em idosos acima de 65 anos $9 \%$, bem como os fatores imunológicos também se encontram relacionados (ABRAMSON JS e GIVNER LB, 1999). 
A taxa de mortalidade desta doença no Brasil relaciona-se à demora do diagnóstico, levando ao comprometimento do sucesso terapêutico. Entre 1989 e 2008 tiveram 737 casos com 186 óbitos, com destaque para região sudeste que apresentou o maior número de casos da doença (BRASIL, 2019).

No que se refere à profilaxia, o uso de antibióticos não oferece vantagens em casos suspeitos por exposição à picada do vetor, isso apenas retardaria o diagnóstico em pacientes assintomáticos, dificultando o tratamento, uma vez que os medicamentos são importantes quando há a comprovação da doença (BRASIL, 2009; KATZ G, et al., 2009). A principal forma de prevenção da FM é evitar áreas com altas infestações de carrapatos, bem como o tratamento de animais hospedeiros, principalmente os domésticos. Dado que ocorra o contato com o vetor, a remoção rápida e correta deste pode evitar a infecção (MARTINS MEP, 2018).

A FM é uma doença de notificação compulsória no Brasil, sendo notificada no Sistema Nacional de Agravos de Notificação (SINAN), por meio do preenchimento da ficha de notificação e investigação de FM, por parte dos profissionais de saúde. Os dados são registrados nos municípios e enviados ao nível estadual e posteriormente ao Ministério da Saúde, configurando-se como importante fonte de informação e vigilância da doença, com vistas à formulação de políticas públicas, medidas preventivas e de controle da morbimortalidade relacionada à enfermidade (BRASIL, 2009).

O levantamento de dados se faz essencial no que se diz respeito ao controle da doença, permitindo o conhecimento do perfil epidemiológico da doença em determinado território, possibilitando assim a estipulação e ampliação de políticas públicas voltadas para a prevenção e tratamento da doença, e sendo assim, esse trabalho tem por objetivo descrever a ocorrência da Febre Maculosa nas macrorregiões do estado de Minas Gerais no período de 2013 a 2017.

\section{MÉTODOS}

\section{Tipo de Estudo}

Trata-se de estudo transversal, descritivo, com delineamento de série temporal e utilização de dados de natureza secundária referentes à ocorrência de casos de Febre Maculosa, da população residente no Estado de Minas Gerais, notificados no Sistema de Informação de Agravo e Notificação (SINAN).

\section{Local e Período da Pesquisa}

Os dados foram coletados por local de residência dos indivíduos cadastrados no SINAN, correspondentes ao período entre os anos de 2013 e 2017. A unidade de análise selecionada para este estudo foi o Estado de Minas Gerais.

\section{População do Estudo, Critérios de Inclusão e Exclusão}

Foram incluídos todos os indivíduos com diagnóstico de Febre Maculosa, residentes no Estado de Minas Gerais, cadastrados no SINAN, no período de 2013 a 2017. Não foram excluídos indivíduos da amostra.

\section{Coleta de dados}

Os dados foram extraídos do SINAN, fornecido pelo Departamento de Informática do Sistema Único de Saúde (DATASUS) e organizados em arquivo de planilhas do softwareMicrosoft ${ }^{\circledR}$ Office Excel versão 15.0, por dois pesquisadores independentes com o propósito de identificar possíveis discrepâncias. Realizou-se uma análise exploratória para reconhecimento das variáveis e correção de possíveis erros ou inconsistências na digitação dos dados. Feitas as correções necessárias precedeu-se a organização e análise dos dados, aplicando-se a estatística descritiva.

\section{Análise dos Dados}

Foram calculadas as proporções dos casos de Febre Maculosa, estratificadas por Macrorregião, discriminadas por sexo, faixa etária, escolaridade, raça/cor, local de residência, zona de infecção, ambiente da infecção, critério de confirmação e evolução expressas em percentual, usando o método direto. Também foi calculada a proporção de mortalidade. 


\section{Aspectos Éticos e Legais da Pesquisa}

O presente estudo envolveu apenas a descrição e análise de dados secundários de população, de domínio público. Nenhuma informação com identificação individual foi obtida para a realização deste estudo.

\section{RESULTADOS}

A amostra do presente estudo foi composta por um total de 112 casos de Febre Maculosa, ocorridos no Estado de Minas Gerais, e notificados no SINAN, no período 2013 a 2017. Maior número de casos foi observado na macrorregião Sudeste com média de 5 casos/ano. Maior incidência média, no período analisado, foi vista na macrorregião do Jequitinhonha de 0,47 casos por 100.000 habitantes. A média de casos no estado foi de 22 casos/ano, com incidência média de 0,11 casos por 100.000 habitantes.

A Tabela 1 apresenta a população e o número de casos registrados por ano, assim como a média, por macrorregião e total.

O cálculo da incidência anual de Febre Maculosa no estado de Minas Gerais demonstrou maiores taxas nas macrorregiões Sudeste e Centro, em todos os anos analisados, configurando o perfil endêmico da doença nessas macrorregiões. No ano de 2017 foi observada a maior incidência média do período estudado, sendo possível notar que nesse ano, um aumento atípico, porém expressivo, do número de casos da doença nas macrorregiões Cento, Leste do Sul e Jequitinhonha contribuíram para esse fenômeno, como pode ser visto na Tabela 2, que apresenta o número de casos e a incidência anual e média, por macrorregião.

Como se pode observar na Tabela 3, maior ocorrência foi observada no sexo masculino $n=94(83,9 \%)$, nas faixas etárias de 20 a 59 anos $n=67(59,8 \%)$, nas escolaridade de até 8 anos de estudo, $n=23(20,5 \%)$, entre pretos e pardos com $n=59(52,7 \%)$, de residência urbana $n=74(66,1 \%)$, com zona de infecção rural $\mathrm{n}=60(45,5 \%)$. Maioria dos casos foi confirmado por exame laboratorial $\mathrm{n}=95(84,8 \%)$, com evolução para cura $n=62(55,4 \%)$. A mortalidade pelo agravo notificado foi de 41 casos, totalizando $36,6 \%$ da amostra (Tabela 4). 


\section{Revista Eletrônica Acervo Enfermagem / Electronic Journal Nursing Collection | ISSN 2674-7189}

Tabela 1- População e número de casos de Febre Maculosa notificados pelo sistema SINAN em Minas Gerais de 2013 a 2017, estratificados por macrorregião e ano.

\begin{tabular}{|c|c|c|c|c|c|c|c|c|c|c|c|c|}
\hline \multirow{5}{*}{$\begin{array}{l}\text { Total de casos } \\
\text { Macrorregião }\end{array}$} & \multicolumn{12}{|c|}{ Ano } \\
\hline & \multicolumn{2}{|l|}{2013} & \multicolumn{2}{|l|}{2014} & \multicolumn{2}{|l|}{2015} & \multicolumn{2}{|l|}{2016} & \multicolumn{2}{|l|}{2017} & \multicolumn{2}{|c|}{ TOTAL } \\
\hline & \multicolumn{2}{|l|}{17} & \multicolumn{2}{|l|}{13} & \multicolumn{2}{|l|}{19} & \multicolumn{2}{|l|}{22} & \multirow{2}{*}{\multicolumn{2}{|c|}{41}} & \multicolumn{2}{|c|}{112} \\
\hline & & & & & & & & & & & \multicolumn{2}{|c|}{ Média } \\
\hline & Pop & $\mathbf{n}$ & Pop & $\mathbf{n}$ & Pop & $\mathbf{n}$ & Pop & $\mathbf{n}$ & Pop & $\mathbf{n}$ & Pop & $\mathbf{n}$ \\
\hline Triângulo do Sul & 744.497 & 0 & 752.948 & 2 & 761.055 & 0 & 768.771 & 0 & 776.099 & 0 & 760.674 & 0,4 \\
\hline Triângulo do Norte & 1.248 .863 & 0 & 1.260 .398 & 0 & 1.271 .462 & 0 & 1.281 .989 & 0 & 1.291 .987 & 0 & 1.270 .940 & 0 \\
\hline Sul & 2.737 .740 & 0 & 2.755 .109 & 1 & 2.771 .764 & 1 & 2.787 .614 & 3 & 2.802 .660 & 1 & 2.770 .977 & 1,2 \\
\hline Sudeste & 1.641 .619 & 7 & 1.651 .433 & 3 & 1.660 .844 & 6 & 1.669 .802 & 8 & 1.678 .307 & 1 & 1.660 .401 & 5 \\
\hline Oeste & 1.243 .409 & 0 & 1.254 .944 & 1 & 1.266 .025 & 1 & 1.276 .557 & 5 & 1.286 .562 & 1 & 1.265 .499 & 1,6 \\
\hline Norte & 1.651 .601 & 0 & 1.661 .130 & 0 & 1.670 .268 & 0 & 1.678 .958 & 0 & 1.687 .219 & 0 & 1.669 .835 & 0 \\
\hline Noroeste & 686.334 & 0 & 691.080 & 0 & 695.638 & 0 & 699.974 & 0 & 704.088 & 0 & 695.423 & 0 \\
\hline Nordeste & 930.004 & 1 & 931.946 & 0 & 933.815 & 2 & 935.587 & 0 & 937.274 & 1 & 933.725 & 0,8 \\
\hline Leste do Sul & 692.406 & 2 & 694.964 & 0 & 697.416 & 1 & 699.751 & 0 & 701.971 & 8 & 697.302 & 2,2 \\
\hline Leste & 1.515 .889 & 1 & 1.523 .095 & 2 & 1.530 .013 & 1 & 1.536 .591 & 1 & 1.542 .840 & 3 & 1.529 .686 & 1,6 \\
\hline Jequitinhonha & 295.924 & 0 & 296.870 & 1 & 297.778 & 1 & 298.645 & 1 & 299.462 & 4 & 297.736 & 1 \\
\hline Centro- Sul & 775.560 & 0 & 780.011 & 0 & 784.283 & 0 & 788.353 & 0 & 792.210 & 1 & 784.083 & 0,2 \\
\hline Centro & 6.429 .510 & 6 & 6.480 .169 & 3 & 6.528 .740 & 6 & 6.574 .968 & 4 & 6.618 .857 & 21 & 6.526 .449 & 8 \\
\hline Total & 20.593 .356 & 17 & 20.734.097 & 13 & 20.869 .101 & 19 & 20.997 .560 & 22 & 21.119 .536 & 41 & 20.862 .730 & 22 \\
\hline
\end{tabular}

Legenda: $n$ - Número de casos de FM.

Fonte: SINAN/DATASUS e IBGE, 2019.

REAEnf/EJNC | Vol. 2 | e2391 | DOI: https://doi.org/10.25248/REAenf.e2391.2020 Página 5 de 10 


\section{Revista Eletrônica Acervo Enfermagem / Electronic Journal Nursing Collection | ISSN 2674-7189}

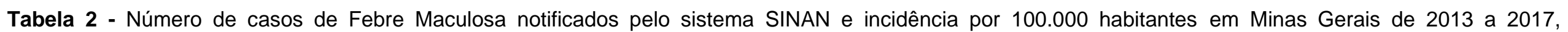
estratificados por macrorregião e ano de ocorrência.

\begin{tabular}{|c|c|c|c|c|c|c|c|c|c|c|c|c|}
\hline \multirow{3}{*}{ Macrorregião } & \multicolumn{12}{|c|}{ Ano } \\
\hline & \multicolumn{2}{|c|}{2013} & \multicolumn{2}{|c|}{2014} & \multicolumn{2}{|c|}{2015} & \multicolumn{2}{|c|}{2016} & \multicolumn{2}{|c|}{2017} & \multicolumn{2}{|c|}{ Média } \\
\hline & $\mathbf{n}$ & Inc & $\mathbf{n}$ & Inc & $\mathbf{n}$ & Inc & $\mathbf{n}$ & Inc & $\mathrm{n}$ & Inc & $\mathbf{n}$ & Inc \\
\hline Triângulo do Sul & 0 & 0,00 & 2 & 0,27 & 0 & 0,00 & 0 & 0,00 & 0 & 0,00 & 0,4 & 0,05 \\
\hline Triângulo do Norte & 0 & 0,00 & 0 & 0,00 & 0 & 0,00 & 0 & 0,00 & 0 & 0,00 & 0 & 0,00 \\
\hline Sul & 0 & 0,00 & 1 & 0,04 & 1 & 0,04 & 3 & 0,11 & 1 & 0,04 & 1,2 & 0,04 \\
\hline Sudeste & 7 & 0,43 & 3 & 0,18 & 6 & 0,36 & 8 & 0,48 & 1 & 0,06 & 5 & 0,30 \\
\hline Oeste & 0 & 0,00 & 1 & 0,08 & 1 & 0,08 & 5 & 0,39 & 1 & 0,08 & 1,6 & 0,13 \\
\hline Norte & 0 & 0,00 & 0 & 0,00 & 0 & 0,00 & 0 & 0,00 & 0 & 0,00 & 0 & 0,00 \\
\hline Noroeste & 0 & 0,00 & 0 & 0,00 & 0 & 0,00 & 0 & 0,00 & 0 & 0,00 & 0 & 0,00 \\
\hline Nordeste & 1 & 0,11 & 0 & 0,00 & 2 & 0,21 & 0 & 0,00 & 1 & 0,11 & 0,8 & 0,09 \\
\hline Leste do Sul & 2 & 0,29 & 0 & 0,00 & 1 & 0,14 & 0 & 0,00 & 8 & 1,14 & 2,2 & 0,32 \\
\hline Leste & 1 & 0,07 & 2 & 0,13 & 1 & 0,07 & 1 & 0,07 & 3 & 0,19 & 1,6 & 0,10 \\
\hline Jequitinhonha & 0 & 0,00 & 1 & 0,34 & 1 & 0,34 & 1 & 0,33 & 4 & 1,34 & 1 & 0,47 \\
\hline Centro- Sul & 0 & 0,00 & 0 & 0,00 & 0 & 0,00 & 0 & 0,00 & 1 & 0,13 & 0,2 & 0,03 \\
\hline Centro & 6 & 0,09 & 3 & 0,05 & 6 & 0,09 & 4 & 0,06 & 21 & 0,32 & 8 & 0,12 \\
\hline Total & 17 & 0,08 & 13 & 0,06 & 19 & 0,09 & 22 & 0,10 & 41 & 0,19 & 22 & 0,11 \\
\hline
\end{tabular}

Legenda: $\mathrm{n}$ - Número de casos de FM; Inc - Incidência.

Fonte: SINAN/DATASUS e IBGE, 2019. 
Tabela 3 - Perfil demográfico da Febre Maculosa no estado de Minas Gerais, no período de 2013 a 2017.

\begin{tabular}{|c|c|c|}
\hline & $\mathbf{n}$ & Proporção \\
\hline Amostra & 112 & $\%$ \\
\hline \multicolumn{3}{|l|}{ Sexo } \\
\hline Masculino & 94 & 83,9 \\
\hline Feminino & 18 & 16,1 \\
\hline \multicolumn{3}{|l|}{ Faixa Etária } \\
\hline Até 19 & 27 & 24,1 \\
\hline 20 à 59 & 67 & 59,8 \\
\hline $60+$ & 18 & 16,1 \\
\hline \multicolumn{3}{|l|}{ Escolaridade } \\
\hline Ignorado/Branco & 64 & 57,1 \\
\hline Até 8 anos de estudo & 23 & 20,5 \\
\hline Ensino Médio Incompleto & 4 & 3,6 \\
\hline Ensino Médio Completo & 7 & 6,3 \\
\hline Ensino Superior Incompleto & 3 & 2,7 \\
\hline Ensino Superior Completo & 2 & 1,8 \\
\hline Não se aplica & 9 & 8,0 \\
\hline \multicolumn{3}{|l|}{ Raça/cor } \\
\hline Ignorado/Branco & 15 & 13,4 \\
\hline Branca & 37 & 33,0 \\
\hline Preta e Parda & 59 & 52,7 \\
\hline Amarela & 1 & 0,9 \\
\hline \multicolumn{3}{|l|}{ Residência } \\
\hline Ignorado/Branco & 8 & 7,1 \\
\hline Urbana & 74 & 66,1 \\
\hline Rural & 29 & 25,9 \\
\hline Periurbana & 1 & 0,9 \\
\hline \multicolumn{3}{|l|}{ Zona Infecção } \\
\hline Ign/Branco & 14 & 12,5 \\
\hline Urbana & 27 & 24,1 \\
\hline Rural & 60 & 45,5 \\
\hline Periurbana & 11 & 9,8 \\
\hline \multicolumn{3}{|l|}{ Ambiente da Infecção } \\
\hline Ignorado/Branco & 18 & 16,1 \\
\hline Domiciliar & 31 & 27,7 \\
\hline Trabalho & 27 & 24,1 \\
\hline Lazer & 28 & 25,0 \\
\hline Outro & 8 & 7,1 \\
\hline \multicolumn{3}{|l|}{ Critério de Confirmação } \\
\hline Ignorado/Branco & 3 & 2,7 \\
\hline Laboratório & 95 & 84,8 \\
\hline Clínico-Epidemiológico & 14 & 12,5 \\
\hline \multicolumn{3}{|l|}{ Evolução } \\
\hline Ignorado/Branco & 7 & 6,3 \\
\hline Cura & 62 & 55,4 \\
\hline Óbito pelo agravo notificado & 41 & 36,6 \\
\hline Óbito por outra causa & 2 & 1,8 \\
\hline
\end{tabular}

Legenda: $\mathrm{n}$ - Número de casos de FM.; Inc - Incidência; RR - Risco relativo.

Fonte: SINAN/DATASUS e IBGE, 2019. 
Alta taxa de mortalidade pela doença foi observada no período, sendo registrados 41 casos de óbito por FM, com taxa de 36,6\% com intervalo de confiança (95\%) de 27,9-46,3\%. A Tabela 4 apresenta a taxa de mortalidade por Febre Maculosa no estado de Minas Gerais.

Tabela 4 - Mortalidade por Febre Maculosa no estado de Minas Gerais no período de 2013 a 2017.

\begin{tabular}{ccc}
\hline $\mathbf{n}$ & Óbitos pelo agravo notificado & $\%$ (IC 95\%) \\
\hline 112 & 41 & $36,6(27,9-46,3)$
\end{tabular}

Legenda: $\mathrm{n}$ - Número de casos de FM; IC - Intervalo de Confiança.

Fonte: SINAN/DATASUS e IBGE, 2019.

\section{DISCUSSÃO}

Na população estudada, observou-se maior ocorrência de FMB no sexo masculino, nas faixas etárias entre 20 e 59 anos e entre pretos e pardos. Esses achados são semelhantes aos encontrados em duas pesquisas, uma realizada no Brasil e outra no Estado de São Paulo, onde houve predominância de casos entre os homens, pretos e pardos e nas faixas etárias de 20 a 59 anos (NASSER JT, et al., 2015; DE OLIVEIRA SV, et al., 2017).

Nos Estados Unidos, estudo realizado também demonstrou maior incidência e risco de mortalidade entre os homens adultos, com maior mortalidade entre os idosos. A maior incidência entre os homens esteve relacionada à exposição laboral em áreas rurais. Entretanto a incidência da FM entre adultos tem diminuído ao longo dos anos e entre crianças tem aumentado, o que tem gerado preocupações das autoridades de saúde no país (DAHLGREN FS, et al., 2012).

O fato de os homens apresentarem maiores chances de morte pode ser explicado por sua exposição mais frequente em ambientes de alto risco e/ou pela tendência de não procurarem atendimento médico em momento oportuno (DAHLGREN FS et al., 2012). Segundo o Ministério da Saúde, o índice de infecção por FM é mais frequente entre homens, que vivem e/ou trabalham em áreas rurais e periurbanas, assim como nas faixas etárias entre 20 a 59 anos devido ser uma população economicamente ativa (BRASIL, 2016).

A idade adulta está relacionada às atividades ocupacionais nas zonas rurais, de ecoturismo, de exploração de matas ou até mesmo na criação de novas moradias, quando se tem contato com a mata e/ou o foco natural da doença, onde os hospedeiros intermediários e o carrapato vetor encontram-se presentes (MONTEIRO KJL, et al., 2014).

Os determinantes de saúde relacionados a raça/cor, têm sido descritos em pesquisas em saúde, com maior vulnerabilidade entre pretos, pardos e indígenas, apresentando níveis mais elevados de adoecimento e de morte por causas evitáveis, incluindo doenças infecto parasitárias, o que pode justificar os achados do presente estudo (LOPES F, 2005).

Quanto ao local de residência, maior ocorrência foi observada em moradores da zona urbana, com $n=74$ $(66,1 \%)$, e zona rural com local fonte de infecção, $n=60$ (45,5\%). Quanto ao ambiente de infecção, trabalho $\mathrm{n}=27(29,1 \%)$, domicílio $\mathrm{n}=31(27,7 \%)$ e lazer foram predominantes. Esses achados podem se justificar uma vez que a transmissão da doença é mais comum nas áreas rurais, entretanto, esse perfil tem se modificado nos últimos anos, e ocorrência de casos em zonas periurbanas e urbanas, tem aumentado, devido à proximidade de pastagens, matas ciliares rios e lagos, principalmente com a presença de equinos e de capivaras (SZABÓ MPJ, et al., 2013).

Pesquisa recente demonstrou que o fato de residir em áreas urbanas esteve associado a menor risco de morte por FA. Esse fato pode estar em parte relacionado ao acesso mais fácil dos habitantes de áreas urbanas aos serviços de saúde. Por outro lado, nas áreas rurais, o parasitismo frequente e comum de carrapatos, pode ser um risco à saúde desconsiderado ou desconhecido por pessoas que residem ou trabalham nesse ambiente (DE OLIVEIRA SV, et al., 2017). 
Destaca-se que os índices de infecções se relacionam com o baixo nível de instrução, pois pessoas que tiveram até 8 anos de estudo foram as mais afetadas, $n=23(20,5 \%)$, e as com nível superior completo, representaram apenas $1,8 \%$ dos casos. Entretanto os fatores envolvidos na vulnerabilidade da população diante da infecção por um agente biológico são múltiplos, incluindo aspectos relacionados à falta de conhecimento e informação, sendo o baixo nível de escolaridade um fator determinante nesses casos. As populações periféricas e rurais também são muito vulneráveis por determinantes sociais e ambientais, incluindo o baixo nível de escolaridade (ARAUJO RP, et al., 2016).

Maior incidência foi observada nas macrorregiões Jequitinhonha $(0,47$ casos $/ 100.000$ habitantes), Leste do Sul ( 0,32 casos/100.000 habitantes) e Sudeste ( 0,30 casos/100.000 habitantes), estando muito acima da média do Estado, no período analisado $(0,11$ casos $/ 100.000$ habitantes). Estudos anteriores têm demonstrado que essas áreas são endêmicas para a doença, e historicamente apresentam maiores incidência (AMÂNCIO FF, et al., 2011).

Entretanto, mostra-se necessário identificar fatores socioeconômicos e agravantes de contaminação, e intervir com medidas de controle, prevenção e conscientização sobre a doença em estudo (LUNA EJA e SILVA JUNIOR JB, 2013).

A proporção de mortalidade no período analisado foi de $36,6 \% \quad(n=112)$, demonstrando queda em relação a períodos anteriormente pesquisados, todavia ainda se apresenta maior que a média nacional (AMÂNCIO FF, et al., 2011; ARAUJO RP, et al., 2016).

Estudos têm demonstrado que a letalidade relacionada à doença está associada a fatores diversos. A infecção pela espécie Rickettsia ricketsii, produz quadros clínicos mais severos, com mortalidade superior a outras espécies (ANGERAMI RN, et al., 2012). As comorbidades associadas, como Diabetes Mellitus, AIDS, neoplasias, doenças pulmonares, coinfecções e imunossupressão também evoluem com maior gravidade (DAHLGREN FS, et al., 2012).

A exposição ao vetor infectado nas suas formas de ninfa e larva aumenta o tempo de exposição e inoculação do patógeno, por serem menos perceptíveis que o carrapato adulto, bem como dificulta o diagnóstico precoce, ocultando o vínculo epidemiológico e consequentemente atraso no tratamento adequado aumentando a mortalidade (KATZ G, et al., 2009).

Os extremos etários, crianças menores de 2 anos e idosos acima de 70 anos, bem a condição de imunidade do hospedeiro, também foram descritos como fatores de risco para maior mortalidade (ABRAMSON JS e GIVNER LB, 1999).

Os casos graves de febre maculosa evoluem com hipotensão, choque, disfunção renal como oligúria e anúria, alterações neurológicas como convulsão, estupor e coma, manifestações hemorrágicas e distúrbios respiratórios. Essas condições clínicas são de alta gravidade e representam risco cinco vezes maior de resultar em morte (CHEN LF e SEXTON DJ, 2008; ANGERAMI RN, et al., 2012).

Como medidas preventivas recomenda-se o uso de repelentes específicos na pele exposta, o tratamento das roupas e equipamentos de trabalho rural com permetrina, o controle de carrapatos nos animais de estimação, o exame da pele e remoção adequada dos carrapatos quando da exposição em áreas rurais onde são encontrados os vetores (PHILLIPS J, 2017).

\section{CONCLUSÃO}

Observou-se maior incidência de casos de Febre Maculosa em pessoas do sexo masculino, com menos de 8 anos de estudos, idade entre 20 e 59 anos, raça parda e negra, residentes nas macrorregiões Jequitinhonha, Leste do Sul e Sudeste, com alta mortalidade no período analisado. Estes dados evidenciam necessidade de atenção do poder público nas áreas mais incidentes, com foco na Atenção Básica, a fim de modificar essa realidade através de campanhas de conscientização, abordando a identificação de sinais e sintomas da doença, medidas de controle de vetores e prevenção da contaminação, a fim de reduzir a ocorrência de casos e principalmente de óbitos. 


\section{REFERÊNCIAS}

1. ABRAMSON JS, GIVNER LB. Rocky Mountain spotted fever. Pediatric Infect Dis J, 1999; 18(6): 539-540.

2. AMÂNCIO FF, et al. Epidemiological characteristics of Brazilian spotted fever in Minas Gerais State, Brazil, 20002008. Cadernos de Saúde Pública, 2011; 27(10): 1969-1976.

3. ARAUJO RP, et al. Febre maculosa no Brasil: estudo da mortalidade para a vigilância epidemiológica. Cad. saúde colet., Rio de Janeiro, 2016; 24(3): 339-346.

4. BRASIL, Ministério da Saúde, Secretaria de Vigilância em Saúde. Guia de vigilância epidemiológica/Ministério da Saúde. 6a ed. Brasília: Ministério da Saúde; 2005;

5. BRASIL, Ministério da Saúde. Secretaria de Vigilância em Saúde. Coordenação-Geral de Desenvolvimento da Epidemiologia em Serviços. Guia de Vigilância em Saúde: [recurso eletrônico] / Ministério da Saúde, Secretaria de Vigilância em Saúde, Coordenação Geral de Desenvolvimento da Epidemiologia em Serviços. - 1. ed. atual. Brasília: Ministério da Saúde; 2016; 773 p.

6. BRASIL, Ministério da Saúde. Tabela de óbitos de febre maculosa. Brasil, Grandes Regiões e Unidades Federadas. 1989- 2009; 2019.

7. CHEN LF, SEXTON DJ. What's new in Rocky Mountain spotted fever? Infect Dis Clin North Am, 2008; 22(3): 415432.

8. DE OLIVEIRA SV, et al. Predictive Factors for Fatal Tick-Borne Spotted Fever in Brazil. Zoonoses and Public Health, 2017; 64(7): e44-e50.

9. DEL FIOL FS, et al. A febre maculosa brasileira. Rev Pan de Salud Púb, 2010; 27(6): 461-466.

10. DIAS E, MARTINS AV. Spotted fever in Brazil. A summary. Am J Trop Med Hyg, 1939; 19(2): 103-108.

11. GUERRANT RL, et al. Tropical Infectious Diseases: Principles, Pathogens, \& Practice. 2nd Edition. Philadelphia: Churchill Livingstone; 2006; 1936p.

12. KATZ G, et al. Situação epidemiológica e importância da febre maculosa no Estado de São Paulo. Bepa, 2009; 6(69):4-13.

13. LUNA EJA, SILVA JUNIOR JB. Doenças transmissíveis, endemias, epidemias e pandemias. In FUNDAÇÃO OSWALDO CRUZ. A saúde no Brasil em 2030 - prospecção estratégica do sistema de saúde brasileiro: população e perfil sanitário [online]. Rio de Janeiro: Fiocruz/lpea/Ministério da Saúde/Secretaria de Assuntos Estratégicos da Presidência da República, 2013; (2): 123-176.

14. LOPES F. Para além da barreira dos números: desigualdades raciais e saúde. Cad Saúde Pública, 2005; 21:1595601.

15. MARTINS MEP. Febre maculosa: revisão-atualização, situação brasileira e goiana. Multi-Science Journal, [S.I.], 2018; $1(5): 15-23$;

16. MONTEIRO KJL, et al. Diagnóstico diferencial entre a febre maculosa brasileira e o dengue no context das doenças febris. Rev Patol Trop, 2014; 43(3): 241-50.

17. NASSER JT, et al. Urbanização da febre maculosa brasileira em município da região Sudeste: epidemiologia e distribuição espacial. Rev Bras Epidemiol, 2015; 18(2): 299-312.

18. ROCKY MOUNTAIN SPOTTED FEVER, United States, 1981. Morbidity and Mortality Weekly Report,31:261-3, 1982

19. SZABÓ MPJ, et al. Ecology, biology and distribuition of spotted-fever tick vectors in Brazil. Front Cell Infect Microbiol, 2013; 3: 27. 